\title{
BISON CONTACT IMPROVEMENTS CASL FY14 REPORT
}

B. W. Spencer, J. D. Hales, D. R. Gaston, D. A. Karpeev, R. L. Williamson, S. R. Novascon, D. M. Perez, R. J. Gardner, K. A. Gamble

September 2014

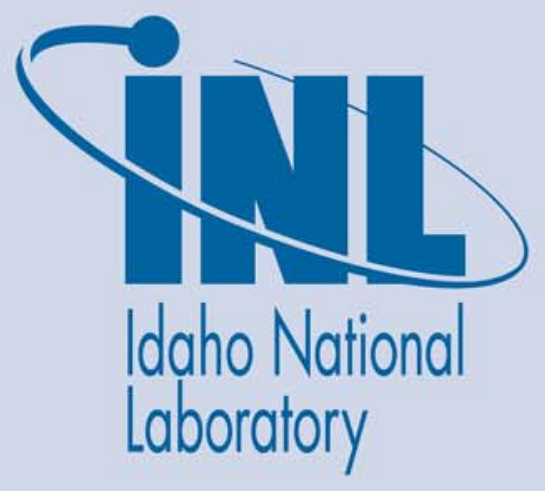

The INL is a U.S. Department of Energy National Laboratory operated by Battelle Energy Alliance 
INL/EXT-14-33285

\title{
BISON CONTACT IMPROVEMENTS CASL FY14 REPORT
}

B. W. Spencer, J. D. Hales, D. R. Gaston, D. A. Karpeev, R. L. Williamson, S. R. Novascon, D. M. Perez, R. J. Gardner, K. A. Gamble

September 2014

\author{
Idaho National Laboratory \\ Idaho Falls, Idaho 83415
}

http://www.inl.gov

Prepared for the

U.S. Department of Energy

Office of Nuclear Science

Under DOE Idaho Operations Office

Contract DE-AC07-05ID14517 


\section{Introduction}

The BISON code is the foundation for multiple fuel performance modeling efforts, including CASL-supported light water reactor fuel model development. The CASL program has supported a number of developments to core fuel modeling capabilities in BISON, as well as developments specifically to the BISON-CASL code, which is based on BISON, with additional models to specifically support CASL efforts. For a variety of fuel forms, the effects of heat conduction across a gap and mechanical contact between components of a fuel system are very significant. It is thus critical that BISON (and BISON-CASL by extension, because the contact code is shared) have robust capabilities for enforcement of thermal and mechanical contact. This report documents efforts supported by the CASL program in FY 2014 to improve the robustness of those contact capabilities.

BISON's solver robustness has generally been quite good before mechanical contact between the fuel and cladding occurs, but there have been significant challenges obtaining converged solutions once that contact occurs and the solver begins to enforce mechanical contact constraints. During the current year, significant development effort has been focused on the enforcement of mechanical contact to provide improved solution robustness.

In addition to this work to improve mechanical contact robustness, an investigation into questionable results attributable to thermal contact has been performed. This investigation found that the order of integration typically used on the surfaces involved in thermal contact was not sufficiently high. To address this problem, a new option was provided to permit the use of a different integration order for surfaces, and new usage recommendations were provided.

\section{Mechanical contact robustness improvements}

The original implementation of mechanical contact enforcement in BISON and MOOSE is based on a system in MOOSE known as the DiracKernel, which is used to apply boundary conditions to a point. For the node on face, master/slave mechanical contact enforcement, two instances of Dirackernel are created: one for the slave side to apply point loads at nodes, and one for the master side to apply point loads at the locations on faces of the nodes on the opposing surface. This system correctly enforces the constraints, but it is limited because it does not provide the ability to fill in all of the off-diagonal entries in the Jacobian matrix used for preconditioning.

In the preconditioned Jacobian-free Newton Krylov (JFNK) algorithm used by BISON to solve the tightly coupled nonlinear equation system, a series of linear iterations are used to obtain each iterative update to the nonlinear solution in Newton's method. If a solution struggles to converge these linear iterations, it is often an indication of a poor preconditioner. That has typically been the case for BISON problems that experienced solution difficulties during contact enforcement. For that reason, the efforts to improve solution robustness with contact have been focused on improving the preconditioner.

A new system in MOOSE known as the Constraint system has been developed to permit improved and more flexible enforcements of arbitrary constraints between arbitrary solution variables at different nodes. The Constraint system can be used to enforce the same mechanical contact constraints that have traditionally been enforced using the DiracKernel system. 
The advantages of the Constraint system are that the same $\mathrm{C}++$ class with code for enforcing contact can be used for both the master and slave sides of the interface, and that it permits the computation of the full set of terms in the Jacobian matrix. Providing a more complete set of these terms is expected to improve preconditioning, and hence, improve the robustness of the linear iterations.

The mechanical contact enforcement code has been re-implemented using the Constraint system. A more complete set of coupling terms has been implemented in this system. Both this new system and the original system are available to the user, and a single input file parameter can be used to switch between these systems. The new Constraint-based mechanical contact enforcement algorithm has been tested on a number of models in the BISON test suite, and has resulted in significantly improved solution robustness in many of these cases. Multiple problems that previously would not converge now run to completion, and run times have been shortened on problems that previously did run to completion. The converged solutions are unchanged with the new system, as it enforces the same mathematical constraints on the equation system.

The preconditioning proces consists of both of creating a matrix that is a good approximation of the Jacobian, and also of a method to apply that preconditioner to the solution. Direct solvers can be used to do this, but iterative solvers are often used for efficient, scalable parallel solutions. The preconditioning matrix calculated with the new contact enforcement algorithm results in greatly improved convergence on small problems where direct solvers are used to apply the preconditioner.

There are still challenges, however, with the iterative methods typically used to precondition larger BISON models. The algebraic multigrid (AMG) preconditioner that has been traditionally used on BISON models often does not converge as well with the Constraint system as it did with the Dirackernel system. It is believed that this is because of the way the AMG algorithm coarsens out degrees of freedom, and the way this interacts with contact nodes. Work is in progress to develop a way to split out the contact degrees of freedom into a separate system and precondition it separately from the rest of the problem. While the AMG preconditioner doesn't work as well with the new system, it has been found that an additive Schwartz method (ASM) works very well on most $2 \mathrm{D}$ problems with the new contact enforcement. This is the standard method currently being used for 2D problems.

The new Constraint system requires more memory usage than the DiracKernal system because of the additional terms in the preconditioner. While that has not been a severe issue for most 2D problems, this has limited its effectiveness for large 3D problems. Work is underway to address this issue by minimizing unnecessary entries in the Jacobian.

In summary, the developments this year have resulted in significant improvements in convergence robustness for $2 \mathrm{D}$ problems, and the new contact enforcement system is being regularly used for 2D models. Developments currently underway are expected to further improve the robustness and efficiency of solutions of models with contact in 2D, and enable the use of the new algorithms on 3D problems, which should provide robustness improvements similar to those already realized for 2D models. 


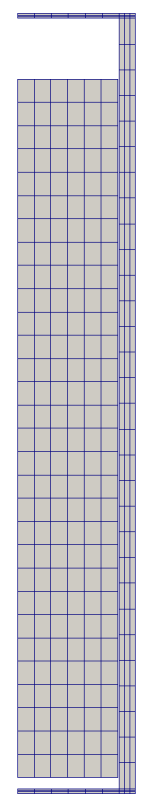

Figure 1: Mesh of LWR fuel rod that is the subject of the investigation on thermal contact behavior. The axial dimension is scaled by a factor of 0.1. QUAD8 elements are used for the fuel and cladding.

\section{Thermal Contact Investigation}

Problems were observed in a BISON-CASL analysis of the smeared LWR fuel rod shown in Figure 1. A rodlet having a length of approximately $0.37 \mathrm{~m}$ was modeled with a smeared pellet mesh with QUAD8 elements. The rodlet was subjected to a uniform axial power, with the history shown in Figure 2.

Because the model has uniform geometry along its axis and uniform boundary conditions along its axial dimension, it was expected that away from the rod ends, the power profile should be essentially uniform. However, as the analysis proceeded, oscillations in the temperature plotted along the axial dimension along the outside of the fuel material began to appear. Figure 3 shows temperature oscillations representative of those that were observed. These temperature oscillations often became so severe that convergence difficulties were encountered, and the model could not run to completion as a result.

After studying this problem and experimenting with various model parameters, it was determined that axial oscillations in the temperature in the fuel adjacent to the cladding were caused by the following phenomena:

- The quadratic interpolation functions used by the QUAD8 elements used in this analysis result in oscillations in the temperature near the ends of the rod. These oscillations are attenuated out quickly away from the ends, and should have a minor effect on the analysis.

- Larger oscillations in the temperature appear later on in the analysis after mechanical contact has been established due to the pressure-dependent term in the gap conductance 


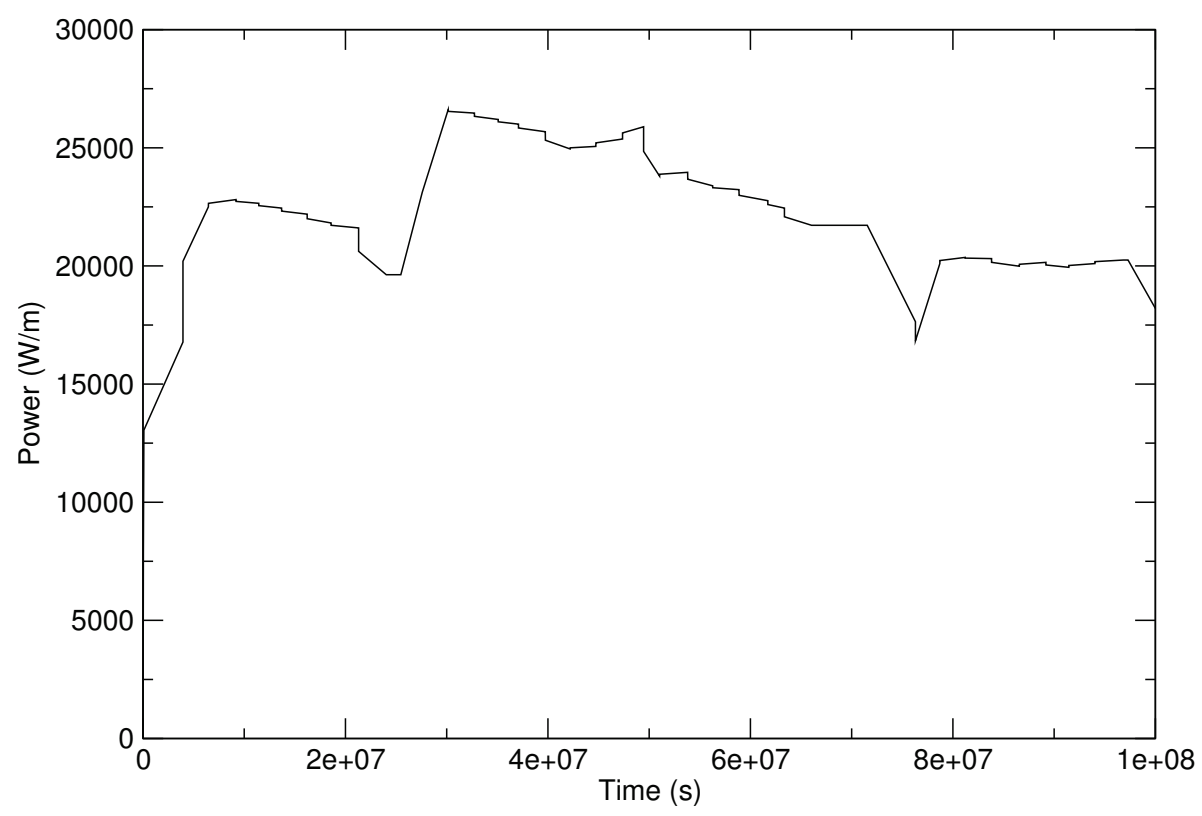

Figure 2: Power history for thermal contact investigation problem.

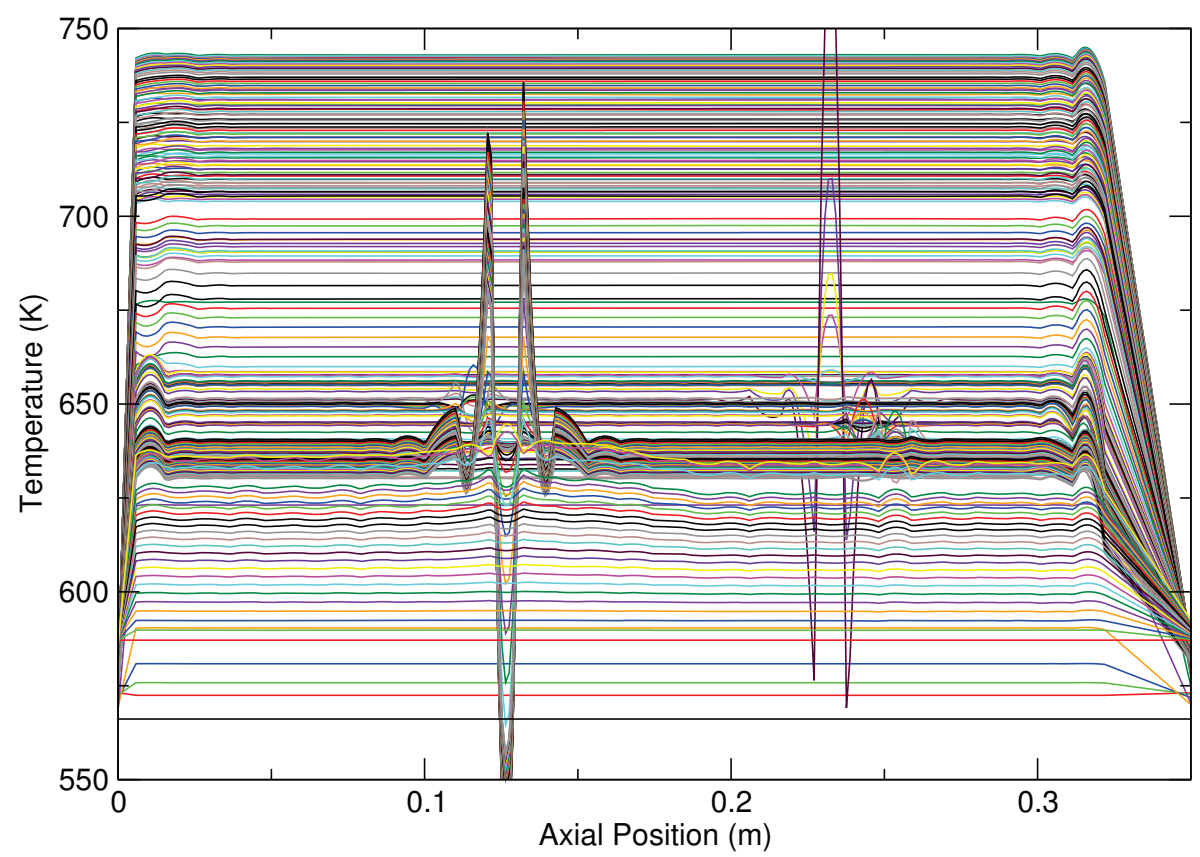

Figure 3: Temperature at fuel outer diameter plotted along rod axis for case with 5th order integration for volumes and 5th order integration for surfaces. 
model. There is some variation in the pressure along the axis due to the variation in the alignment of the elements on opposing sides of the fuel/pellet contact interface. The temperature osciallations due to this effect grow larger with increased contact pressure. These completely go away if the pressure-dependent term in the gap conductance is disabled.

- The large, random oscillations shown in Figure 3 are due to instabilities in the solution caused by an insufficiently high integration on the surface. These are the most problematic of the types of oscillations discussed here because they can lead to seemingly random nonphysical swings in the temperature and solution failure.

The default integration order for QUAD8 elements is a 5th order Gauss scheme, which uses a $3 \times 3$ array of integration points in each elements. Prior to this investigation, MOOSE always used the same integration scheme for volume and surface elements, so the 5 th order scheme used 3 integration points per element on the surface. Figure 3 shows the results of using the default 5 th order scheme on the volume and surface. Because the power history reaches a maximum and then decreases over time, the temperature profiles start out at low values, increase to a maximum, and then gradually decrease over time. Late in time, the model with these settings starts to exhibit extremely large swings in the temperature. It is important to emphasize that all results shown here are for fully converged solutions.

There is clearly an instability in the thermal solution. This is similar to "hourglass" instabilities that can be observed in solid mechanics solutions with reduced integration elements due to the zero energy modes that occur with an insufficiently high order integration scheme if steps are not taken to suppress those zero energy modes. While the 5th order integration scheme of the thermal fluxes on the contact surfaces should be sufficiently high order to not have zero energy modes, local problems can occur due to the way elements on opposing sides of the interface align with each other.

To test the theory that the errors were due to an instability caused by unsufficiently high order integration of the fluxes on the contact surfaces, the same model was run with a 7 th order integration rule. A $4 \times 4$ grid of integration points was used on volumetric elements, and 4 points were used on each element surface. With this higher order integration rule, the previouslyobserved instabilities completly went away. This is not a very optimal solution to the problem, however, becase the memory and computational time requirements are significantly higher with more integration points.

To achieve stable solutions without an undue penalty to the solution time, a new option was added to MOOSE to permit the user to specify the order of integration on surfaces independently of the integration order for volumes. Figure 4 shows the axial temperature profile obtained using the default 5th order integration scheme on the volume together with the 7 th order integration scheme on the surfaces. These results are essentially identical to the results obtained using 7 th order integration for both surface and volume.

BISON has commonly been run using reduced-order integration with QUAD8 elements. Instead of using the 5 th order scheme, a 3rd order scheme, with a $2 \times 2$ grid of integration points is used on the volume elements. This integration scheme was tested in conjunction with various integration schemes on the surfaces to see if stable solutions could be achieved. Figure 5 shows the temperature profiles for a case with 3 rd order integration in the volume and 7 th order inte- 


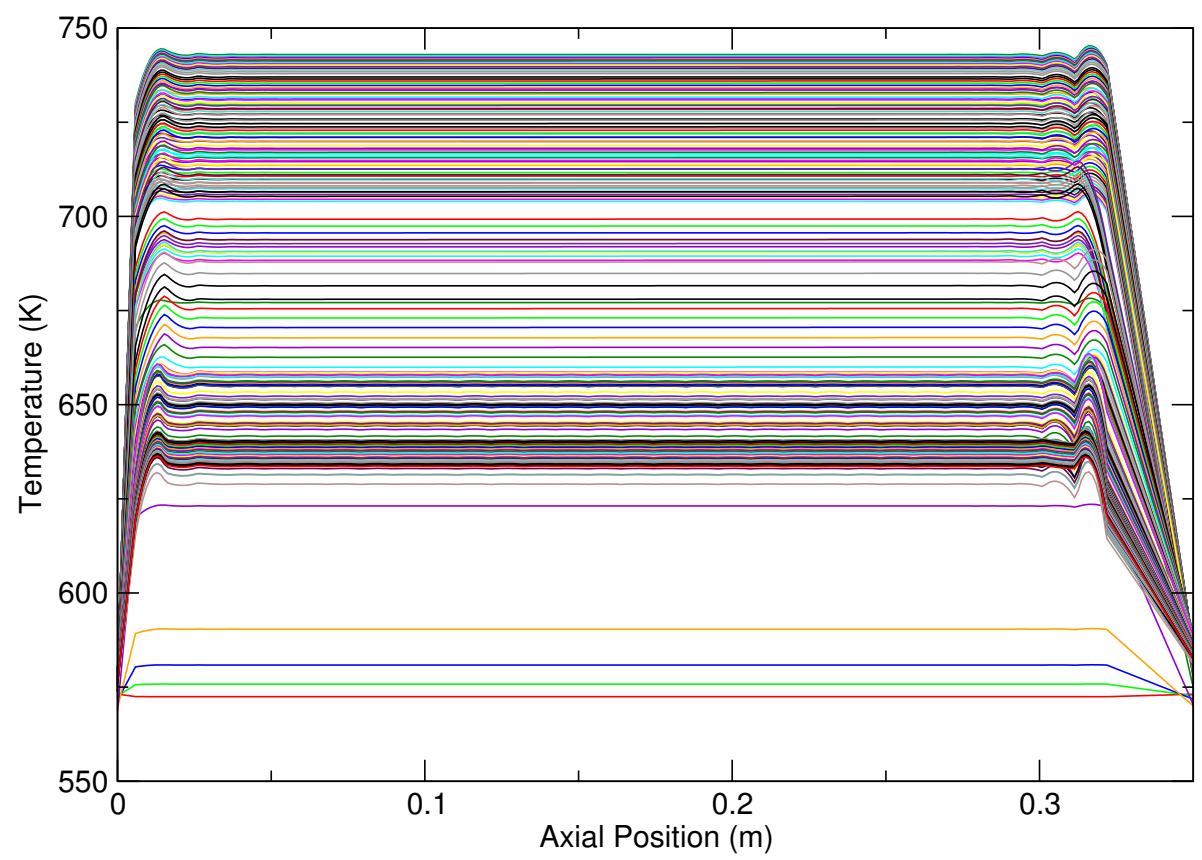

Figure 4: Temperature at fuel outer diameter plotted along rod axis for case with 5th order integration for volumes and 7th order integration for surfaces.

gration on the surface. Large oscillations and instabilities are clearly evident, indicating that this scheme should not be used.

The results shown here are all for QUAD8 elements. For QUAD4 elements, stable solutions were only obtained with at least a 3rd order scheme in the volume elements and a 5th order scheme on the surfaces. The conclusion of this work is thus that with the thermal contact enforcement used in MOOSE/BISON, for solution stablity, full integration must be used for volumetric elements, and one level higher than full integration must be used for integrated boundary conditions on surfaces.

It should be noted that full integration can result in volumetric locking in solid mechanics problems in some circumstances. For 3D problems, the solid mechanics models in MOOSE employ a scheme that uses reduced integration for the pressure to avoid this problem. A similar capability for 2D problems should be developed as well.

Based on the results of this study, the suite of BISON validation problems has been converted to use these new recommendations. The robustness and validity of these models was improved significantly as a result, and this is the current recommendation.

\section{Summary and Future Work}

As summarized in this report, the contact-related work conducted during the current fiscal year has resulted in significant improvements in both robustness and accuracy of thermal and mechanical contact in BISON. Switching 2D models over to use the new Constraint system for 


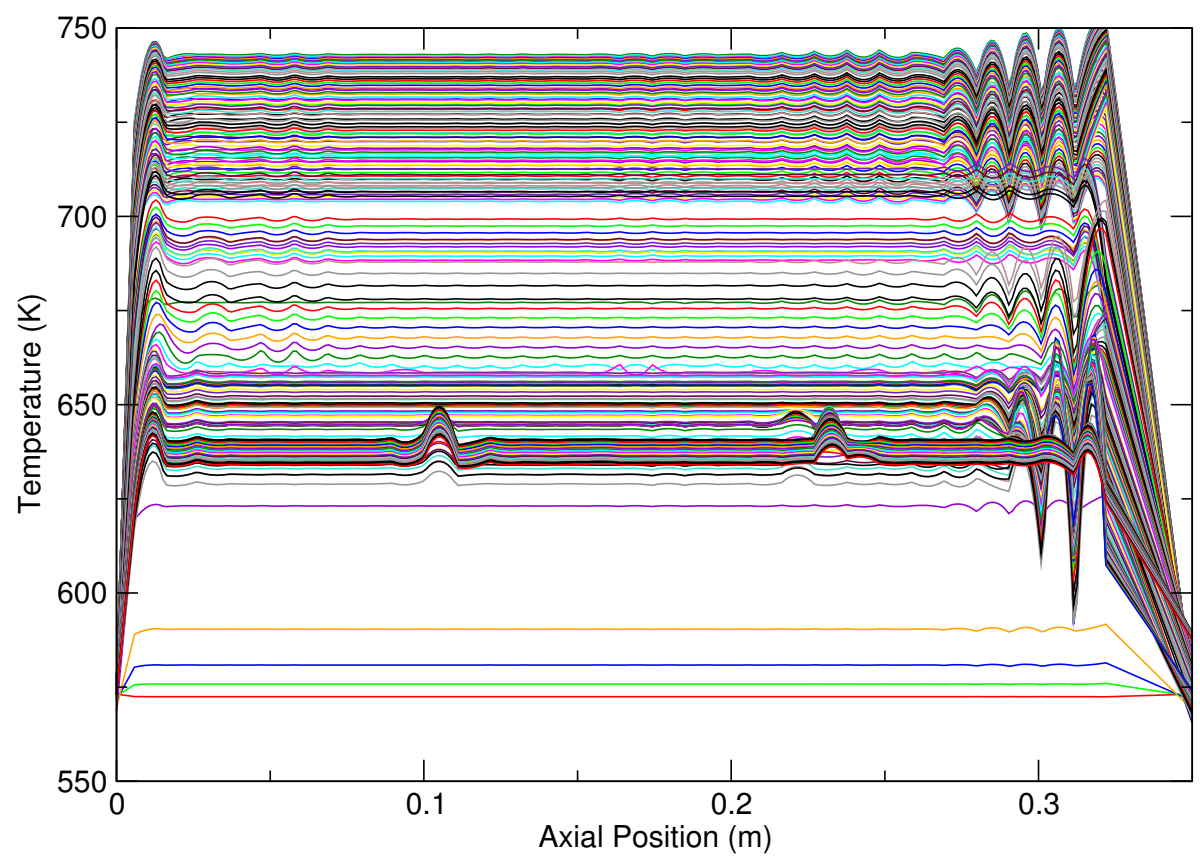

Figure 5: Temperature at fuel outer diameter plotted along rod axis for case with 3rd order integration for volumes and 7th order integration for surfaces.

mechanical contact enforcement has consistently resulted in more robust convergence when appropriate preconditioning options are used. Using the newly-developed option to specify higher order integration for faces gives better results and convergence for thermal contact.

While this work has resulted in significant improvements, there is still more work to do in this area. Work is in progress on several fronts to reduce the memory usage with the Constraint system. The set of interacting nodes is being reduced to the minimal size, and options are being tested to supply a reduced set of Jacobian entries. In addition, work is underway to test code that partitions the system of equations into a part with all contact nodes, and a part with the remainder of the system. This will hopefully permit the usage of more efficient preconditioning options.

The usage of higher order quadrature for the integration of thermal contact surfaces appears to resolve the observed stability issues. In the future, mortar-based methods for contact enforcement under development will better integrate thermal contact under a variety of conditions, and the higher-order integration scheme currently used will not be necessary. 